\title{
Massless QED on Random-Block Lattice
}

Ting-Wai Chiu ${ }^{\text {* }}$

a Department of Physics, National Taiwan University, Taipei, Taiwan 106, R.O.C.

Massless QED is regularized using random-block lattice ( RBL ). Using naive fermion without explicitly breaking chiral symmetry or gauge symmetry in the action, it is shown that physical observables can avoid the effects of species doubling after averaging over an ensemble of RBL. For 2D QED, numerical results of dynamical fermion simulations using Hybrid Monte Carlo are in good agreement with the exact solutions of the Schwinger model in continuum.

\section{INTRODUCTION}

In this paper, I report the present status of the ongoing project of using an ensemble of randomblock lattices to provide a proper measure for functional integrals involving fermion fields. The readers are referred to [1] for introduction and earlier results.

\section{Massless QED on RBL}

The action of massless QED for single flavor of the fermion field on a RBL is

$$
\begin{array}{r}
A_{r b l}=\sum_{i, \mu} \omega_{i} K_{i}^{\mu} \bar{\psi}_{i} \gamma_{\mu}\left[U_{\mu}(i) \psi_{i+\hat{\mu}}-U_{\mu}^{\dagger}(i-\hat{\mu}) \psi_{i-\hat{\mu}}\right] \\
+\beta \sum_{p} w_{p}\left[1-\operatorname{Re}\left(U_{p}\right)\right]
\end{array}
$$

where $\psi_{i}$ and $\bar{\psi}_{i}$ are two independent spinors at site $i, U_{\mu}(i)$ is the link variable pointing from the site $i$ to the site $i+\hat{\mu}$,

$U_{\mu}(i)=\exp \left[\operatorname{iel}_{i}^{\mu} A_{\mu}\left(x_{i}+\frac{1}{2} l_{i}^{\mu}\right)\right], \quad l_{i}^{\mu}=x_{i+\mu}-x_{i}$

$\omega_{i}$ is the site weight of the site $i, K_{i}^{\mu}$ is the inverse of the distance between the sites $i+\hat{\mu}$ and $i-\hat{\mu}$,

$\omega_{i}=\prod_{\mu} \frac{\left(x_{i+\hat{\mu}}-x_{i-\hat{\mu}}\right)}{2}$,

$$
K_{i}^{\mu}=\left(x_{i+\hat{\mu}}-x_{i-\hat{\mu}}\right)^{-1}
$$

"This work is supported by the National Science Council, R.O.C. under the grant number NSC86-2112-M002-017.
The $\gamma$ matrices are chosen in 2D to be $\gamma_{1}=\sigma_{1}$, $\gamma_{2}=\sigma_{2}$ and $\gamma_{5}=-i \gamma_{1} \gamma_{2}=\sigma_{3}$, while in 4D

$$
\begin{aligned}
\gamma^{i} & =\left(\begin{array}{cc}
0 & -i \sigma^{i} \\
i \sigma^{i} & 0
\end{array}\right), \quad i=1,2,3 \\
\gamma_{4} & =\left(\begin{array}{ll}
0 & 1 \\
1 & 0
\end{array}\right) \\
\gamma_{5} & =\gamma_{1} \gamma_{2} \gamma_{3} \gamma_{4}=\left(\begin{array}{cc}
1 & 0 \\
0 & -1
\end{array}\right)
\end{aligned}
$$

The last term in the action $A_{r b l}$ is the kinetic energy of the gauge field which is conveniently labeled as

$$
A_{g}[U]=\beta \sum_{p} w_{p}\left[1-\operatorname{Re}\left(U_{p}\right)\right]
$$

where $\beta=\frac{1}{e^{2}}, U_{p}$ is the path-ordered product of the link variables around a plaquette $p, w_{p}$ is the weight of the plaquette $p$, which is the ratio of the area dual to $p$ and the area of $p$,

$w_{p}=\frac{\tilde{S_{p}}}{S_{p}}, \quad S_{p}=l_{i}^{\mu} l_{i}^{\nu}, \quad \tilde{S_{p}} S_{p}=\prod_{\lambda=1}^{D} l_{i}^{\lambda}$

The action $A_{r b l}$ is gauge invariant as well as chiral invariant. After integrating out the fermion field, the partition function becomes

$Z=\int[d U] \operatorname{det}(G) \exp \left(-A_{g}[U]\right)$

where $G$ is the matrix

$G_{i j}^{\alpha \beta}=\gamma_{\mu}^{\alpha \beta} \omega_{i} K_{i}^{\mu}\left[U_{\mu}(i) \delta_{j, i+\hat{\mu}}-U_{\mu}^{\dagger}(i-\hat{\mu}) \delta_{j, i-\hat{\mu}}\right]$ and $\operatorname{det}(G)$ is positive definite. In $2 \mathrm{D}, G$ can be written as

$G=\left(\begin{array}{cc}0 & T_{1}-i T_{2} \\ T_{1}+i T_{2} & 0\end{array}\right)$ 
where $T_{1}$ and $T_{2}$ are anti-hermitian matrices. Then $\operatorname{det} G=\operatorname{det}\left(K^{\dagger} K\right)>0$, where $K \equiv T_{1}+$ $i T_{2}$. In $4 \mathrm{D}, G$ can be written as

$G=\left(\begin{array}{cc}0 & -K^{\dagger} \\ K & 0\end{array}\right)$

where

$K=\left(\begin{array}{cc}T_{4}+i T_{3} & T_{2}+i T_{1} \\ -T_{2}+i T_{1} & T_{4}-i T_{3}\end{array}\right)$

and $T_{i}, i=1, \ldots 4$ are anti-hermitian matrices. Again we have $\operatorname{det} G=\operatorname{det}\left(K^{\dagger} K\right)>0$. Therefore the probability distribution function is positive definite for any gauge field configurations and thus amenable for Monte Carlo simulations even for one flavor of the fermion field. Complex scalar fields $\left\{\phi_{i}, \phi_{i}^{*}\right\}$ are introduced to convert $\operatorname{det}(G)$ to a functional integral over the complex scalar fields. Then the partition function becomes

$$
\begin{array}{r}
Z \simeq \int[d U]\left[d \phi^{\dagger}\right][d \phi] \exp \left(-A_{g}[U]-\phi^{\dagger}\left(K^{\dagger} K\right)^{-1} \phi\right) \\
\simeq \int[d U]\left[d \xi^{\dagger}\right][d \xi] \exp \left(-A_{g}[U]-\xi^{\dagger} \xi\right)
\end{array}
$$

where $\xi \equiv\left(K^{\dagger}\right)^{-1} \phi$. Then the system is equivalent to that of effective action

$$
A_{e f f}=A_{g}[U]+\xi^{\dagger} \xi
$$

in which quantum expectation value of any observables can be computed by Hybrid Monte Carlo ( HMC ) simulation [2]. The procedures of performing $\mathrm{HMC}$ are similar to those on a hypercubical lattice.

\section{OBSERVABLES}

For 2D QED, measurements of the Wilson loop and the current correlation function at $\beta=3.0$ and $\beta=10.0$ were performed using an ensemble of 64 random-block lattices, each of size $12 \times 12$ and average lattice spacing $a=1.0$. The fermion fields satisfy antiperiodic boundary conditions while the gauge links satisfy the periodic boundary conditions. On each RBL, 10,000 sweeps are used for thermalization, 20,000 measurements were performed with consecutive measurements separated by 10 sweeps. The ensemble averages are in good agreement with the exact solutions of Schwinger model [3] on a torus [4]. In the following, only the current correlation function is discussed. In continuum, the current correlation function is defined by

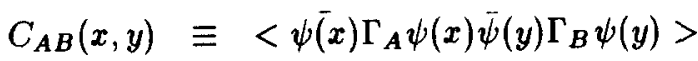

$$
\begin{aligned}
& =\frac{1}{Z} \int[d A][d \bar{\psi}][d \psi] \exp \left(-A_{\mathcal{C}}\right) \\
& (-1) \operatorname{tr}\left[S_{F}(x, y) \Gamma_{B} S_{F}(y, x) \Gamma_{A}\right]
\end{aligned}
$$

where $\Gamma_{A}$ and $\Gamma_{B}$ are any two matrices in the spinor space, and $S_{F}(x, y)=G^{-1}(x, y)$ is the fermion propagator. Since the integrand involves product of local operators, the gauge invariant point-splitting is used to regularize the singularities at the end points $x$ and $y$ respectively. Thus we have

$$
\begin{array}{r}
C_{A B}(x, y, \epsilon)=\frac{1}{Z} \int[d A][d \bar{\psi}][d \psi] \exp \left(-A_{c}\right) \\
\times F_{A B}(x, y, \epsilon)
\end{array}
$$

where

$$
\begin{aligned}
& F_{A B}(x, y, \epsilon)=\frac{-1}{4} \operatorname{tr}[ \\
& S_{F}\left(x+\frac{\epsilon}{2}, y-\frac{\epsilon}{2}\right) \Gamma_{B} S_{F}\left(y+\frac{\epsilon}{2}, x-\frac{\epsilon}{2}\right) \Gamma_{A} P(x) P(y) \\
& +S_{F}\left(x+\frac{\epsilon}{2}, y+\frac{\epsilon}{2}\right) \Gamma_{B} S_{F}\left(y-\frac{\epsilon}{2}, x-\frac{\epsilon}{2}\right) \Gamma_{A} P(x) P^{*}(y) \\
& +S_{F}\left(x-\frac{\epsilon}{2}, y-\frac{\epsilon}{2}\right) \Gamma_{B} S_{F}\left(y+\frac{\epsilon}{2}, x+\frac{\epsilon}{2}\right) \Gamma_{A} P^{*}(x) P(y) \\
& \left.+S_{F}\left(x-\frac{\epsilon}{2}, y+\frac{\epsilon}{2}\right) \Gamma_{B} S_{F}\left(y-\frac{\epsilon}{2}, x+\frac{\epsilon}{2}\right) \Gamma_{A} P^{*}(x) P^{*}(y)\right]
\end{aligned}
$$

and the phase factor $P(z)$ is the path-ordered product

$$
P(z)=\exp \left[\mathrm{ie} \int_{z-\frac{e}{2}}^{z+\frac{\varepsilon}{2}} A \cdot d l\right]
$$

To regularize $C_{A B}(x, y, \epsilon)$, an ensemble of RBL is generated with 4 fixed sites, $x-\frac{\epsilon}{2}, x-\frac{\epsilon}{2}, y-\frac{\epsilon}{2}$, and $y+\frac{\epsilon}{2}$. Then the functional integral is replaced by the multiple integrals on each RBL. Since each RBL is equally probable, the final result must be averaged over all RBL. The RBL regularized current correlation function is

$<C_{A B}(x, y, \epsilon)>\simeq \frac{1}{N_{\text {latt }}} \sum_{l=1}^{N_{\text {latt }}} C_{A B}^{l}(x, y, \epsilon)$ 
where $C_{A B}^{l}(x, y, \epsilon)$ is the current correlation function measured on the $l-t h$ random-block lattice,

$$
\begin{array}{r}
C_{A B}^{l}(x, y, \epsilon)=\frac{1}{Z} \int[d U][d \xi]\left[d \xi^{\dagger}\right] \exp \left(-A_{e f f}\right) \\
\times F_{A B}(x, y, \epsilon)
\end{array}
$$

where the phase factor $P(z)$ is replaced by path ordered product of link variables connecting $z-\frac{\epsilon}{2}$ and $z+\frac{\epsilon}{2}$. All possible choices of $\Gamma_{A}$ and $\Gamma_{B}$ have been computed. For the chiral correlation function, $\Gamma_{A}=\left(1+\gamma_{5}\right) / 2, \Gamma_{B}=\left(1-\gamma_{5}\right) / 2$, it is plotted in Fig. 1, for an ensemble of $64 \mathrm{RBL}$ each of size $12 \times 12$ and average lattice spacing $a=1.0$. It agrees with the Schwinger model on the torus. Other observables are also in good agreement with the continuum solutions. They will be reported elsewhere.

\section{CONCLUSIONS and DISCUSSIONS}

The present investigation of dynamical fermion on RBL is far from completed yet. Nevertheless, the present numerical results of $2 \mathrm{D}$ vector gauge theory are consistent with the conclusions drawn from previous studies [1] of free fermion and fermion in a background gauge field, that an ensemble of RBL could provide a proper measure for functional integrals involving fermion fields. Without breaking the chiral symmetry or gauge symmetry at the tree level, RBL regularization applying to the naive fermion which presumably suffers from the species doubling on any one of the lattices, however miraculously gives the correct result when the functional integrals are sumed over all RBL. One of the difficulties of using RBL regularization is that we must perform independent measurements on each lattice of the ensemble, yet the number of lattices to gaurantee good ensemble averages is not known a priori. This extra factor $\left(10^{3} \sim 10^{4}\right)$ of computing time imposes a serious obstacle to use RBL for any $4 \mathrm{D}$ realistic computations on the present computing platforms. Nevertheless it would be interesting to find out whether RBL works for anomaly free chiral gauge theories.

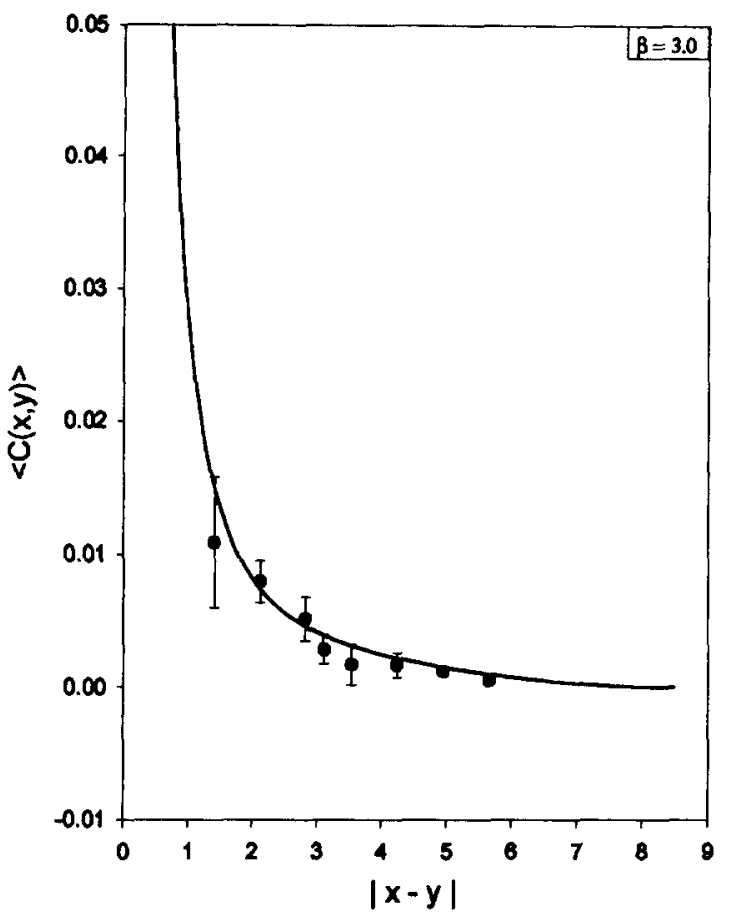

Figure 1. The chiral correlation function $<C(x, y)>$ plotted vs. $|x-y|$ at $\beta=3.0$. The end points $x$ and $y$ are both at the diagonal with the point-splitting $\epsilon=(0.7,0.7)$. The solid line corresponds to the Schwinger model on the torus of the same size.

\section{REFERENCES}

1. T.W. Chiu, Phys. Lett. B 206 (1988) 510; Phys. Lett. B 217 (1989) 151; Nucl. Phys. B ( Proc. Suppl.) 34 (1994) 599; Nucl. Phys. B ( Proc. Suppl.) 42 (1995) 603; Nucl. Phys. B ( Proc. Suppl.) 53 (1997) 647.

2. S. Duane, A.D. Kennedy, B.J. Pendleton and D. Roweth, Phys. Lett. B 195 (1987) 216.

3. J. Schwinger, Phys. Rev. 128 (1962) 2425.

4. I. Sachs and A. Wipf, Helv. Phys. Acta. 65 (1992) 652; Y.C. Kao, Mod. Phys. Lett. A 7 (1992) 1411. 